\title{
Clinic, Diagnosis and Treatment of Somatic (Somatized) Depression
}

NA Aliyev ${ }^{1 *}$ and ZN Aliyev ${ }^{2}$

${ }^{1}$ Azerbaijan State Advanced Training Institute for Doctors named by A.Aliyev, department of psychi $\neg$ atry and drug addiction, Baku, Azerbaijan

${ }^{2}$ Azerbaijan Medical University, department of psychiatry Baku, Azerbaijan

\section{"Correspondence author}

\section{Dr. Nadir A Aliyev}

Department of Psychiatry and Addiction Azerbaijan State Advanced Training Institute for Doctors named by A. Aliyev

Department of Psychiatry and Addiction

Baku

Azerbaijan

Submitted : 12 March 2020 ; Published : 20 April 2020

\begin{abstract}
Objective: Somatic depression consisted of the development of appropriate practical recommendations, new treatment methods that consistently present the clinical, diagnostic and therapeutic aspects of depression.

Materials and Research Methods: The study was conducted at the Mental Health Center of the Ministry of Health on January 1, 2015, from January 1, 2020, for 100 patients with somatic depression. The clinical and psychopathological method was mainly used to detect somatized depression.

Results: Conclusion Masked depression is present in 75\% of pains (sore throat) and various disorders in different parts of the body. In these cases, 55\% suffer from discomfort and functional disturbances in the gastrointestinal tract.

Conclusion: Daily change of state, worse in the evening and early morning, relief at noon; periodic, wavelike and spontaneous somatic and mental disorders; seasonality of somatic and mental disorders, most often in the autumn-spring period; ineffective somatic treatment and the positive effect of antidepressant therapy;
\end{abstract}

Keywords: Somatic Depression, Clinic, Diagnosis and Therapy

\section{Introduction}

The World Health Organization (WHO) conference in Geneva on October 9, 2012, dedicated to the 20th anniversary of World Mental Health Day (October 10), called for an end to the stigmatization of those suffering from depression and other mental disorders [1]. Depression is the most common mental disorder. More than 350 million people, including all age groups, suffer from depression. The WHO likens depression to an epidemic. Depression is the number one cause of absenteeism and the second leading cause of disability. If appropriate measures are not taken, the depression in 2020 will paralyze the economies of both developed and developing countries. Today, Sweden ranks first and the United States second in terms of depression-related illness.
Somatic depression is a very important part of mental disorders. Patients with this diagnosis are treated for a long time by various doctors - surgeons, interns, neurologists - without any positive results. At the same time, effective care for these patients is possible only after a correct diagnosis by a psychiatrist and continuous treatment with antidepressants for months. He purpose of the study. Somatic depression has consisted of the development of appropriate practical recommendations and new treatment methods, with a consistent presentation of clinical, diagnostic and therapeutic aspects.

\section{Materials and Methods}

The study was conducted at the Mental Health Center of the 
Ministry of Health on 100 patients suffering from somatic depression from January 1, 2015, to January 1, 2020. The clinical-psychopathological method was mainly used to detect somatic depression. It should be noted that despite the fact that all patients have been treated by different doctors for many years, there are no positive results. They were sent to the Mental Health Center of the Ministry. Of the 100 people examined, 70 were women and 30 men, or $70 \%$ were women.

Table 1: General characteristics of the examined patients

\begin{tabular}{|l|l|}
\hline Female / Male 70/30 & $\mathbf{7 0 / 3 0}$ \\
\hline Age group & $30-55$ \\
\hline Education: & 50 \\
\hline Higher Education & 30 \\
\hline Secondary Vocational & 20 \\
\hline Secondary & $60 / 40$ \\
\hline Employment Status & \\
\hline Works / Doesn't work & 50 \\
\hline Marital Status & 20 \\
\hline She/he is married & 30 \\
\hline She/he is single & \\
\hline She/he is a widow &
\end{tabular}

The following classification was used to describe the results obtained in the study of somatic depression [2]:

1. Algig forms of somatic depression

1.1. Abdominal-gastrointestinal discomfort and functional disorders

1.2. Cardiac - sensory and autonomic disorders of the cardiovascular system.

1.3. Cephalgia - with the predominance of headaches.

1.4. Panaligig - a large number of polymorphic and variable unpleasant sensations in different parts of the body.

2. Acripnia with various sleep disorders and persistent insomnia.

3. Diencephalic with the predominance of autonomic disorders:

3.1. Vegetovisseral - vegetative disorders are an episodically generalized (functionalhypothalamic) type of vegetative crisis (crisis).

3.2. Symptoms of vasomotor rhinitis are vasomotorallergic.

3.3. Pseudo-asthmatic with choking seizures.

4. Obsessive-phobic, characterized by the predominance of painful flashing thoughts, memories, doubts, fears.

5. Drug addiction - abuse of psychoactive substances, including alcohol, during the depression.

\section{Results Obtained and Their Discussion}

The results of the study of somatic depression in the examined patients were given in Table 2

Table 2: Frequency of different types of somatic depression

\begin{tabular}{|c|c|c|}
\hline S.No & Types of somatic depression & Number \\
\hline 1 & $\begin{array}{l}\text { Pain (algig) forms of somatic } \\
\text { depression }\end{array}$ & 75 \\
\hline 1.1 & $\begin{array}{l}\text { Abdominal-gastrointestinal discomfort } \\
\text { and functional disorders }\end{array}$ & 55 \\
\hline 1.2 & $\begin{array}{l}\text { Cordial - sensory and autonomic } \\
\text { disorders of the cardiovascular system }\end{array}$ & 7 \\
\hline 1.3 & $\begin{array}{l}\text { Cephalgia - with the predominance of } \\
\text { headaches }\end{array}$ & 8 \\
\hline 1.4 & Glossalgia & 1 \\
\hline 1.5 & Arthralgia & 1 \\
\hline 1.6 & $\begin{array}{l}\text { Panaligig - a large number of } \\
\text { polymorphic and variable unpleasant } \\
\text { sensations in different parts of the body }\end{array}$ & 3 \\
\hline 2 & $\begin{array}{l}\text { Acripnia with various sleep disorders } \\
\text { and persistent insomnia }\end{array}$ & 5 \\
\hline 3 & \multicolumn{2}{|l|}{$\begin{array}{l}\text { Diencephalic with the predominance of } \\
\text { autonomic disorders: }\end{array}$} \\
\hline 3.1 & $\begin{array}{l}\text { Vegetovisseral - vegetative disorders are } \\
\text { the episodically generalized (functional- } \\
\text { hypothalamic) type of vegetative crisis } \\
\text { (crisis) }\end{array}$ & 5 \\
\hline 3.2 & $\begin{array}{l}\text { Symptoms of vasomotor rhinitis are } \\
\text { vasomotor-allergic }\end{array}$ & 4 \\
\hline 3.3 & Pseudo-asthmatic with choking seizures & 5 \\
\hline 4 & $\begin{array}{l}\text { Obsessive-phobic, characterized by } \\
\text { the predominance of painful flashing } \\
\text { thoughts, memories, doubts, fears }\end{array}$ & 4 \\
\hline 5 & $\begin{array}{l}\text { Narkomanig- abuse of psychoactive } \\
\text { substances, including alcohol, during } \\
\text { depression }\end{array}$ & 2 \\
\hline Total & & 100 \\
\hline
\end{tabular}

Somatic depression, in relation to borderline mental disorders, differs from gross disorders of the psyche - psychoses and other mental disorders, which are engaged in major psychiatry. This field is small (it is not small in the true sense of the word), so before psychiatrists, there are field therapists, pediatricians, broad-profile doctors. What is known about how patients perceive depression, what symptoms it manifests and how it can disappear?

\section{Mental and Somatic Balance}

It is clear that somatic depression manifests itself as a link between "pure" mental disorders (including endogenous classical depression) and psychosomatic diseases (stomach, 
duodenal ulcer, asthma, hypertension, etc.). But unlike somatic diseases, in somatic depression the disorder is reversible, i.e. the process is functional. Often, somatic depression "remains" after a typical (classic) depression in the past. Classical depression is characterized by mood swings, associative, motor inhibition, and changes in somatic tone. If the mental symptoms of depression decrease, motor inhibition disappears, and incomplete (reduced) depression is observed. In this case, the center of gravity of depression falls on the somatic sign in the clinical picture of the disorder and comes to the fore with physical pain and unpleasant feelings; the mental component (mood) goes into the background. In this case, we are talking about depression, disguised as somatic disorders and thus somatic. Finally, somatic disorders completely cover the clinical picture of depression, and the mental component (depression) is no longer understood by patients, making depression extremely difficult to detect or undetected. These conditions are called non-depressive depression.

No matter how much the somatic depression is masked by facades, suicide attempts often occur, despite the depth of the mental component (depression). In essence, the milder the severity of depression, the more intense the somatic manifestations. On the contrary, depression replaces the expressive somatic component (imitation of somatic symptoms).

Thus, psychosomatic balance is observed in somatic depression. Krasnushkin EK, According to him, it is a general law for mental and somatic diseases [3]. A psychosomatic condition such as depression seems to balance - pole in one of the classic depression, somatic equivalent to the other pole (shown) stops. The transition of the psych depressor to the expressive somatic symptoms occ Somatic masks that hide depression

It is known that in the clinical picture of somatic depression, the symptoms of both functional and organic disorders of the body can be imitated. Typical depressive symptoms are "pale", there are numerous and nonspecific somatic disorders. In general, the most characteristic symptoms are a combination of the following: insomnia, loss of appetite, headaches, complaints of disorders of internal organs, and finally, internal anxiety, fatigue. What are the most common masks of depression?

\section{The Variant of the Somatic Mask is Algic-Senestopathic}

In masked depression, pain (alkyl) and various unpleasant sensations in different parts of the body occur in $75 \%$ of cases. $55 \%$ of them have unpleasant sensations and functional disorders of the gastrointestinal tract. That is why this mask of depression is called algic-senestopathic. The pain often begins suddenly; the topographic pain syndrome does not correspond to the innervation zones. Analgesics do not have a positive effect. Patients usually associate the occurrence of pain with the occurrence of an external event, often looking for external causes. Frustration, gloom, lethargy, depression, despair, hopelessness, low mood. Such patients are observed by interns for a long time when they come to a psychiatrist for consultation and treatment and even undergo surgery. Patients also undergo numerous meaningless examinations of the gastrointestinal, cardiovascular and respiratory systems. Despite the fact that patients receive long-term outpatient and inpatient, sanatorium-spa treatment, there is no significant improvement in their condition.

\section{Abdominal Syndrome - Mask}

This is specifically a variant of the algic-senestopathic mask - pain, spasm, burning sensation in the abdomen, numbness, pain, pressure (paraesthesia), etc. It is observed. Patients feel "heaviness" in the stomach, "overfilling", "bursting", "vibration", and "bloating" in the intestines, nausea, and painful cramps. The pain is usually long-lasting, wheezing, incessantly dull, but periodically it can be short-lived and lightning-fast. Such pains are usually periodic (usually more intense at night and in the morning), and usually do not depend on food intake and its nature. They usually lose their appetite, have poor appetite, lose weight, suffer from painful constipation, and rarely have diarrhea. In addition to the pain characteristic of these conditions, flatulence - bloating, bloating, constipation are observed. Patients repeatedly call an ambulance; take them to hospitals on suspicion of gastrointestinal diseases. They are usually diagnosed with gastritis, cholecystitis, pancreatitis, colitis, gastric and duodenal ulcers, biliary dyskinesia, appendicitis, dysbacteriosis, and in some cases, they undergo surgery, but the estimated diagnosis is not confirmed. The results of objective examinations are normal. Importantly, the treatment of the alleged somatic disease is ineffective.

\section{Glossalagia Syndrome - A Mask}

Glossalgia means burning, stinging, itching, numbness, etc. of the tongue and oral cavity observed with pain, feelings are meant. Manifestations of depressive symptoms (depression, anxiety, etc.) are similar to the symptoms of concomitant dental disease, and even for the consultant and the patient, it is difficult to know which is the first and which is the second. However, many objective examinations do not reveal objective changes. In this case, late detection of depression, the patient undergoes orthopedic and dental surgery. It is at the insistence of patients that healthy teeth are removed, even all the teeth of the upper and lower jaw. Most patients complain of the enlarged tongue, difficulty speaking, fatigue when swallowing, the heaviness of the tongue, as if the tongue is perceived as a foreign body, bitterness in the mouth, metallic taste. The pain is relieved in the morning and intensifies in the evening. At the same time, patients often complain of insomnia, nausea, discomfort in the gastrointestinal tract, loss of appetite, pain in various parts of the body (head, heart, abdomen, joints). Such a variety of symptoms does not fit into the framework of any dental disease; its persistent and peculiar course gives rise to suspicion of masked depression. 
Cardiac Syndrome - A Mask Somatic Masks That Hide Depression

It is known that in the clinical picture of somatic depression, the symptoms of both functional and organic disorders of the body can be imitated. Typical depressive symptoms are "pale", there are numerous and nonspecific somatic disorders. In general, the most characteristic symptoms are a combination of the following: insomnia, loss of appetite, headaches, complaints of disorders of internal organs, and finally, internal anxiety, fatigue. What are the most common masks of depression?

\section{The Variant of the Somatic Mask is Algic-Senestopathic}

In masked depression, pain (alkyl) and various unpleasant sensations in different parts of the body occur in $75 \%$ of cases. 55\% of them have unpleasant sensations and functional disorders of the gastrointestinal tract. That is why this mask of depression is called algic-senestopathic. The pain often begins suddenly; the topographic pain syndrome does not correspond to the innervation zones. Analgesics do not have a positive effect. Patients usually associate the occurrence of pain with the occurrence of an external event, often looking for external causes. Frustration, gloom, lethargy, depression, despair, hopelessness, low mood. Such patients are observed by interns for a long time when they come to a psychiatrist for consultation and treatment and even undergo surgery. Patients also undergo numerous meaningless examinations of the gastrointestinal, cardiovascular and respiratory systems. Despite the fact that patients receive long-term outpatient and inpatient, sanatorium-spa treatment, there is no significant improvement in their condition.

\section{Abdominal Syndrome - Mask}

This is specifically a variant of the algic-senestopathic mask - pain, spasm, burning sensation in the abdomen, numbness, pain, pressure (paraesthesia), etc. It is observed. Patients feel "heaviness" in the stomach, "overfilling", "bursting", "vibration", and "bloating" in the intestines, nausea, and painful cramps. The pain is usually long lasting, wheezing, incessantly dull, but periodically it can be short-lived and lightning-fast. Such pains are usually periodic (usually more intense at night and in the morning), and usually do not depend on food intake and its nature. They usually lose their appetite, have a poor appetite, lose weight, suffer from painful constipation, and rarely have diarrhea. In addition to the pain characteristic of these conditions, flatulence - bloating, bloating, constipation is observed. Patients repeatedly call an ambulance; take them to hospitals on suspicion of gastrointestinal diseases. They are usually diagnosed with gastritis, cholecystitis, pancreatitis, colitis, gastric and duodenal ulcers, biliary dyskinesia, appendicitis, dysbacteriosis, and in some cases, they undergo surgery, but the estimated diagnosis is not confirmed. The results of objective examinations are normal. Importantly, the treatment of the alleged somatic disease is ineffective.

\section{Glossalgia Syndrome - A Mask}

Glossalgia means burning, stinging, itching, numbness, etc. of the tongue and oral cavity observed with pain. Feelings are meant. Manifestations of depressive symptoms (depression, anxiety, etc.) are similar to the symptoms of concomitant dental disease, and even for the consultant and the patient, it is difficult to know which is the first and which is the second. However, many objective examinations do not reveal objective changes. In this case, late detection of depression, the patient undergoes orthopedic and dental surgery. It is at the insistence of patients that healthy teeth are removed, even all the teeth of the upper and lower jaw. Most patients complain of the enlarged tongue, difficulty speaking, fatigue when swallowing, the heaviness of the tongue, as if the tongue is perceived as a foreign body, bitterness in the mouth, metallic taste. The pain is relieved in the morning and intensifies in the evening. At the same time, patients often complain of insomnia, nausea, discomfort in the gastrointestinal tract, loss of appetite, pain in various parts of the body (head, heart, abdomen, joints). Such a variety of symptoms does not fit into the framework of any dental disease; its persistent and peculiar course gives rise to suspicion of masked depression.

\section{Cardiac Syndrome - A Mask \\ An Option for a Somatic Mask - Insomnia}

In somatic depression, sleep disturbance is the leading or only symptom. Stubborn, prolonged insomnia (agripnia) does not result in early awakening (usually three-fourths in the morning), shortening of sleep at night, and the effect of hypnotics. Sleep disorders are common in all variants of somatic depression, but their intensity and expression vary. The course of the disease is a period: 5 or more seizures occur in 1-2 days. The first seizure usually begins with a shortterm headache, migraine-like type, manifested as vegetativevascular paralysis, "azan" pain. The longer the disease lasts, the more intense the sleep disorders. The light periods between seizures last from a few months to several years, during which time the person becomes completely healthy. In the morning, patients experience general malaise, drowsiness, heaviness in the head, loss of appetite ("does not even want to think about eating"). The most difficult situation for patients is the first race of the day. By the middle of the day, the general condition improves the appetite increases, the ability to work increases. Depression is assessed by patients, and others associate it with insomnia.

\section{The Variant of the Somatic Mask is Diencephalic}

The hypothalamic (diencephalic) area of the brain is responsible for the complex and varied manifestations of somatic depression. Patients are treated for a long time by a therapist, neurologist, endocrinologist with ineffective treatment with diagnoses of neurocirculatory dystonia, cardioneurosis, somatogenic neurosis, neurasthenia, pancreatitis, asthmatic bronchitis, vasomotor rhinitis. Periodic recurrence of the disorder, the ineffectiveness of therapy, is the basis for referral 
to a psychiatrist. The disease is characterized by pain, insomnia, sexual desire (libido) and decreased potency. In addition to the somatic mask, depressive mood swings, which are not very obvious, are observed within 1-2 days, usually without any external cause (mostly in the spring-autumn period). At this time, unreasonable grief, sorrow, depression ("everything falls out of my hands", "the world has lost its beauty", "I want to be alone", the idea of the meaninglessness of life, the feeling of guilt.

The disease usually begins abruptly at night or early in the morning, waking up at three or four o'clock from an internal shock. The first subjective sign is a blow, a sway, a shake as if the ground is underfoot. This condition is suddenly replaced by tremors, chills, bloating of the whole body, increased heart rate. There is a sharp pain in the heart area, a feeling of cardiac arrest, freezing, irregularities (heartbeat, etc.), sweating, and fear of death.

The seizure usually begins gradually, followed by a cold, chills. Seizures are often seasonal, lasting from 1-2 years to 6 months. Occasionally, nasal congestion, difficulty breathing, and unpleasant sensations are also observed during the light period. Patients are examined and treated by an ENT doctor for a long time (5 or more years) with a diagnosis of hemorrhoids, pancreatitis, allergic polyposis, chronic rhinitis, and even undergo surgery. But no positive effect. Pseudo-asthmatic syndrome against the background of mild depressive disorder Periodically, the frequency, rhythm, and depth of breathing change. Subjectively, patients experience painful suffocation, a feeling of sore throat, shortness of breath. These symptoms are not related to physical exertion and emotional stress. , patients do not have a forced posture, the appearance of patients, the expression of the eyes does not change, the color of the skin and mucous membranes is normal. There are no objective signs of organic disease of the respiratory tract.

\section{The Variant of the Mask - Obsessive-Phobic Syndrome}

Thoughts and fears come to the fore, but at the same time, they are perceived as symptoms of the disease and are mandatory. The appearance of depression is less noticeable, it is a mental mask of depression - a combination of thoughts and fears with depressive symptoms, a special character. In most cases, depression begins in the form of diencephalic crises, which is why patients are often under the supervision of neurologists, therapists, endocrinologists with various diagnoses (neurocirculatory dystonia, myocardial infarction, thyrotoxicosis) and are denied after the examination. Only when there is an expressive fear is a psychiatrist referred, either independently or on the basis of a referral from a specialist. In some, some features of the character appear before the onset of the disease: anxiety, meticulousness, suspicion, skepticism, indecision, indecision, lack of confidence in their own power when making decisions, and so on. It is observed. As a predisease period, sleep disorders, unexplained, transient pain without an organic basis, pain in the heart, abdomen, and head are noted. Sometimes the patient has a history of a depressive episode.

\section{The Variant of the Mask - Drug Addict}

This masked depression is one of the most unpleasant and socially significant. We are talking about a contingent of people suffering from somatic depression, the most common of which is the abuse of psychoactive substances. The fact is that the main manifestation of masked depression (the main disease) is the abuse of alcohol and other psychoactive substances in the form of seizures. That is why addiction to psychoactive substances occurs against the background of periodic depressive and somatic-vegetative disorders. Prior to the formation of this social mask, patients experience anxiety, tension, mood swings, loss of energy and activity, difficulty in contact with others, grief, sadness, unwillingness to live. These conditions occur either for no reason or after mental trauma, but with daily variability, the vital nature is noted. Long before the abuse of psychoactive substances, patients experience a variety of unpleasant pains (heart, stomach), insomnia. Patients are diagnosed with rheumatism, gastric ulcer, vegetative-vascular dystonia, cardiac neurosis, neurasthenia.

Somatic pathology is not detected during the examination and the diagnosed diagnoses are denied, somatic depressive disorders are not understood, these conditions are explained by physical dysfunction. Addiction to psychoactive substances develops quickly because the disease itself - depression - has not been identified. Abstinence syndrome occurs when the use of psychoactive substances is stopped during the somatic depression. In these patients, abstinence syndrome, along with various symptoms, depressive mood - depression, guilt. Self-blame, suicide, suicide, somatovegetative manifestations (various pains, unpleasant feelings, loss of appetite, increased blood pressure, weight loss, dry tongue, increased sweating, etc.) are observed. However, early depression is predominant here. Unlike the somatic mask, in addition to the treatment of depression, changes in the internal organs and nervous system as a result of the abuse of psychoactive substances are required for a long time and the patient is isolated for treatment. Selfblame, suicide, suicide, somatovegetative manifestations (various pains, unpleasant feelings, loss of appetite, increased blood pressure, weight loss, dry tongue, increased sweating, etc.) are observed. However, early depression is predominant here. Unlike the somatic mask, in addition to the treatment of depression, changes in the internal organs and nervous system as a result of the abuse of psychoactive substances are required for a long time and the patient is isolated for treatment.

\section{The Variant of the Mask - Sexual Disorders}

As sexual disorders accompany depression, these disorders are considered to be one of the earliest symptoms of somatic depression. Along with the increase in depressive symptoms, changes in the sexual sphere deepen the duration of sexual intercourse, the expression of an adequate erection in the morning, orgasm decreases, the number of libidos and pollutions decreases, and sexual activity in general. This situation is 
perceived by patients as a tragedy. Sexual dysfunction is central to somatic depression. Sexual abuse hinders marriage, which in turn negatively affects family relationships and, in some cases, leads to divorce. In this case, psychotherapy is not treated with male hormones, but with antidepressants. That is why they associate depression with a failed sexual act. Depression, which is not felt by patients, reduces the energy potential and disrupts the sexual sphere.

\section{Discussion}

At the present stage, the treatment of depression faces several problems. First, because the clinic and course of depression have undergone major changes (pathomorphosis). Second, only $10 \%$ of depression manifests itself in the classical clinical form. Depression often has an atypical, somatic, semi-acutely prolonged form, which cannot be identified and treated in a timely manner. Third, choosing between antidepressants and many new antidepressants in the treatment of depression is a major challenge for professionals. Fourth, resistance to treatment of depression has increased, sometimes due to inadequate prescribing of antidepressants. Fifth, among both previous and new antidepressants, it was found to be effective in treating diseases other than depression. Sixth, there has been recent progress in studying the mechanism of action of antidepressants and the pathogenesis of depression. Earlier in our book, we briefly described the clinic of somatized depression [4]. Thus, it needs further study of the mechanisms of mental and somatic balance, as well as the clinic of somatic depression.

\section{Conclusion}

Advice recommendation

1. A slight decrease in mood, inability to enjoy life as before, difficulty in contact with others, loneliness, a decrease in energy, activity, difficulties in decision-making;

2. Various pains and unpleasant sensations that are difficult to describe, the absence of organic disorders that explain the patient's complaints;

3. The nature of sleep disorders, it is shortening, early awakening, loss of appetite, weight loss, menstrual disorders in women, decreased potency in men;

4. Daily change of the situation, aggravation in the evening and early morning, relief in the afternoon;

5. Periodic, wavy and spontaneous occurrence of somatic and mental disorders;

6. The nature of the season of somatic and mental disorders, mostly in the autumn-spring period;

7. Ineffectiveness of somatic treatment and the positive effect of therapy with antidepressants.

We want to end the topic with a very brief clinical observation: a 28-year-old woman who has been treated by various specialists for more than 10 years. But no positive results were obtained. When he approached us, he had surgery to remove his thyroid gland, the upper third of his stomach, his gallbladder, and finally his spleen. We treated him with maprotiline for 6 months. Catamnestic data show that the woman is married and has two children.

\section{The limitation of our work:}

1. A small number of patients;

2. A short time observation.

Thus, for a group of examined patients with somatic depression in more than 90 percents of cases, is characterized by different variants of somatic masks. The study has a great theoretical and practical importance. First, it will contribute to the early detection of somatic depression. Second, prevent suicides. Third, it will help reduce refractory to treatment of somatic depression.

\section{Author Disclosure Information}

The authors declare that the article is submitted on behalf of all authors. None of the material in the article has been published previously in any form and none of the material is currently under consideration for publication elsewhere other than noted in the cover letter to the editor. Authors declare no financial and personal relationship with other people or organizations that could inappropriately influence this work. All authors contributed to and have approved the final article. The authors declare no conflicts of interest. No sponsor provided funding for this study.

\section{Conflict of interest statement}

The authors declare that the research was conducted in the absence of any commercial or financial relationships that could be construed as a potential conflict of interest.

\section{Acknowledgment}

The authors would like to thank staff of the Mental Health Center of the Ministry of Health of the Republic of Azerbaijan.

\section{References}

1. WHO (2012) Information Bulletin $\mathrm{N}^{\circ} 369$.

2. Desyatnikov VF (1981) Hidden depression in the practice of doctors / V.F. Desyatnikov, TT Sorokina. - Minsk: Higher School 1981: 240.

3. Krasnushkin EK (1960) About some relationships between mental and somatic diseases (selected diseases). M 1960: 427-445.

4. Aliyev NA, Aliyev ZN (2015) Application of antidepressants in psychiatric practice (textbook). Baku, Globe-Print LLC 2015: 322.

Copyright: (C2020 NA Aliyev. This is an open-access article distributed under the terms of the Creative Commons Attribution License, which permits unrestricted use, distribution, and reproduction in any medium, provided the original author and source are credited. 